\title{
CDISC SDTM Frequency Terminology
}

National Cancer Institute

\section{Source}

National Cancer Institute. CDISC SDTM Frequency Terminology. NCI Thesaurus. Code C71113.

The terminology subset that includes terms pertaining to frequency scheduling of interventions and medications within the Clinical Data Interchange Standards Consortium (CDISC) Study Data Tabulation Model (SDTM). 